\title{
CD82 Aggravates Sevoflurane - Induced Neurotoxicity by Regulating TRPM7 in Developing Neurons
}

\author{
Qing Shu ${ }^{1}$, Xiaoyan Zhao ${ }^{1}$, Xin Geng ${ }^{1}$, Xiaoye Wang ${ }^{2, *}$
}

\author{
${ }^{1}$ Department of Pharmacy, Ninth \\ Hospital of Xi'an, Xi'an City, Shaanxi \\ Province, 710054, P. R. China \\ 2 Department of Teaching Research, \\ Ninth Hospital of Xi'an, Xi'an City, \\ Shaanxi Province, 710054, P. R. China
}

\section{*Correspondence}

wangxiaoye67068@126.com

(Xiaoye Wang)

\begin{abstract}
Background: Sevoflurane, a commonly used anesthetic in neonatal, could induce neurotoxicity in newborn animals. CD82 was found to be involved in age-related cognitive impairment. However, the role of CD82 in sevoflurane-induced neurotoxicity remains elusive. Methods: Hippocampal neurons were isolated from neonatal rats (postnatal day 1 or 2), and then exposed to $1.8 \%$ sevoflurane for $6,12,24$ or 48 hours. Neurons were pre-transfected with siRNA targeting CD82 (siCD82) or co-transfected with siTRPM7 (transient receptor potential melastatin 7) and pcDNA 3.1-CD82, and then exposed with sevoflurane $(1.8 \%, 12$ hours). Cell viability of the neurons was analyzed with MTT assay, and cell apoptosis was determined by flow cytometry. Protein expression was analyzed by western blot. Results: Sevoflurane exposure decreased cell viability of the developing hippocampal neurons in a time-dependent manner. Protein expressions of CD82 and TRPM7 were increased in neurons post sevoflurane exposure in a time-dependent manner. Pre-transfection of siCD82 attenuated sevoflurane-induced decrease in cell viability and increase in cell apoptosis in the neurons. Moreover, knockdown of CD82 reversed the promoting effects of sevoflurane on protein expression of cleaved TRPM7 and cleaved caspase-3. Over-expression of CD82 aggravated sevoflurane-induced decrease in cell viability and increase in cell apoptosis in neurons, while knockdown of TRPM7 counteracted with the effects of CD82 over-expression on sevoflurane-induced developing neurons. Conclusion: Sevoflurane exposure increased the expression of CD82 and TRPM7 in developing hippocampal neurons, decreased cell viability and promoted the cell apoptosis. Knockdown of CD82 partially ameliorated sevoflurane-induced neurotoxicity by down-regulation of cleaved TRPM7 in the developing neurons.
\end{abstract}

\section{Keywords}

CD82, TRPM7, Sevoflurane, Neurotoxicity, Neurons

\section{Introduction}

Sevoflurane, isoflurane, ketamine and other drugs are the most common anesthetics in surgical operations [1]. However, mounting studies have found that anesthetics could induce long-term neurotoxicity and cause cognitive impairment in infant brains $[1,2]$. Sevoflurane was reported to induce neuronal apoptosis and abnormal protein deposition, resulting in disruption of cerebral homeostasis and cognitive impairment [3]. Moreover, sevoflurane could also alter metabolomic profiles in neonatal rats and induce neurotoxicity and neurobehavioral dysfunction [4]. Therefore, therapeutic strategies should be developed to protect against sevoflurane anesthesia-induced neurotoxicity in the developing brains.

CD82, a member of tetraspanins, is expressed in most eukaryotes and regulate cell adhesion, cell signaling and membrane trafficking [5]. CD82 was found to associate with cis with $\alpha 4$ integrin to mediate cell adhesion in hematopoietic stem/progenitor cell [5]. CD82 was also found to be involved in tumor cell migration and invasion [6]. Recently, CD82 has been found to negatively regulate the oligodendrocyte development and myelination [7]. In addition, CD82 was reported to be a potential biomarker for patients with central nervous system involvement [8]. Since CD82 could promote secretion of beta-amyloid peptide, thereby mediating age-related cognitive impairment [9], the effect of CD82 on sevoflurane-induced neurotoxicity was then evaluated in this study.

Transient receptor potential melastatin (TRPM7) is widely distributed in periphery and central nervous system and could regulate cell proliferation, death, differentiation and migration [10]. In the nervous system, TRPM7 is involved in a key neurobiological process from synaptic transmission to cognitive function under physiological conditions [11], thereby mediating neurotoxicity, nerve injury and neuronal death [11]. For example, TRPM7 functions as a zinc influx channel, and 


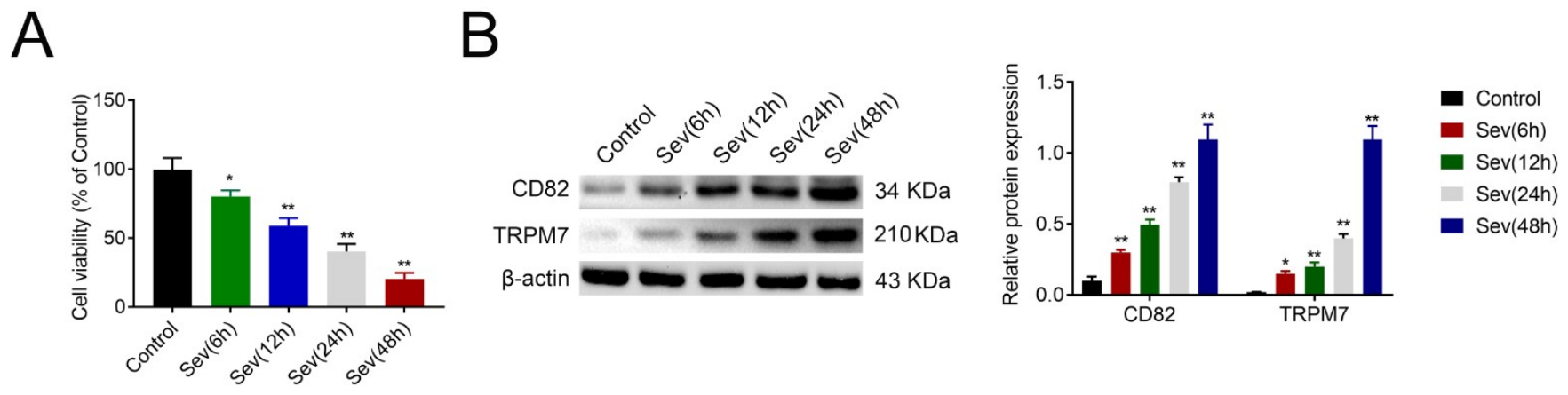

F I G U R E 1. Expression of CD82 and TRPM7 in sevoflurane-induced neurons.

(A) Cell viability of hippocampal neurons was decreased time dependently by sevoflurane exposure.

(B) Protein expression of CD82 and TRPM7 were increased time dependently by sevoflurane exposure. ${ }^{*} p<0.05,{ }^{* *} p<0.01$.
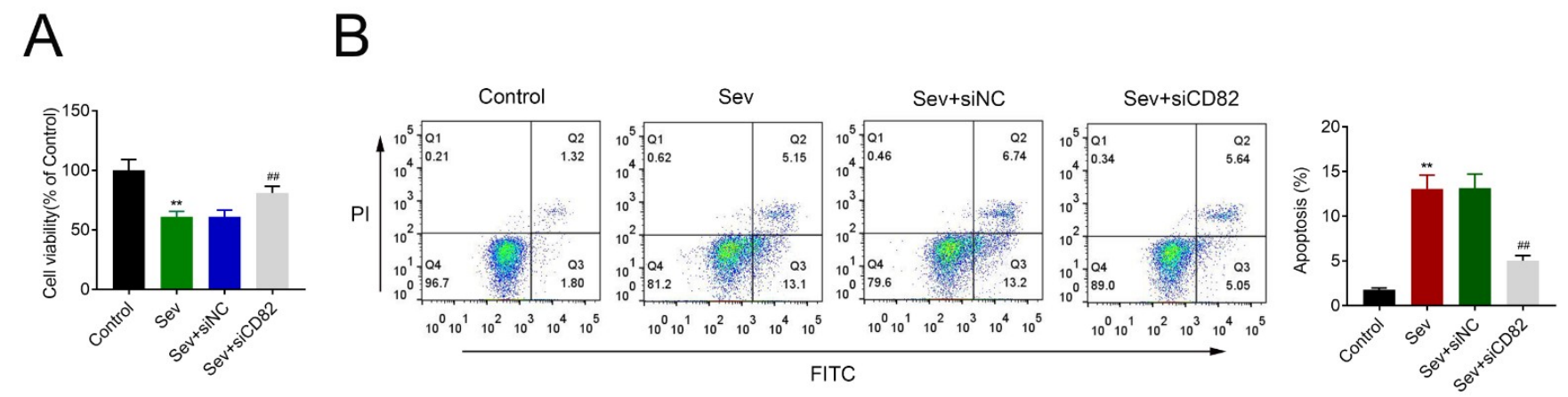

F I G U R E 2. Knockdown of CD82 attenuated sevoflurane-induced neurotoxicity.

(A) Pre-transfection with siCD82 attenuated sevoflurane-induced decrease in neuronal viability.

(B) Pre-transfection with siCD82 attenuated sevoflurane-induced increase in neuronal apoptosis. ${ }^{* *}, \#$ \# $<0.01$.

potentiates the neurotoxicity induced by $\mathrm{Zn}^{2+}$ [12]. Inhibition of TRPM7 could demonstrate neuroprotective effect against hypoxic-ischemic injury in neonatal neurons [13]. Moreover, previous study has shown that CD82 could activate caspase3 to cleave TRPM7, thereby promoting the phosphorylation of Numb to participate in beta-amyloid peptide secretion [9]. Therefore, this study hypothesized that CD82 might promote cleavage of TRPM7 to mediate sevoflurane-induced neurotoxicity.

This study was performed to examine the protein expression of CD82 and TRPM7 in hippocampal neurons after sevoflurane treatment. Additionally, the role of CD82/TRPM7 on sevoflurane-induced neurotoxicity in hippocampal neurons was also determined.

\section{Methods}

\subsection{Isolation and culture of neurons}

All animal experiments were approved by the Biomedical Ethics Committee of Ninth Hospital of Xi'an (Approval no.2020-046) for the use of animals and conducted in accordance with the National Institutes of Health Laboratory Animal Care and Use Guidelines. Neonatal Sprague-Dawley rats at day 1 or day 2 were acquired from Laboratory Animal Center of Tongji Medical College (Wuhan, China). Rats under anesthesia were sacrificed via cervical dislocation. The brain tissues were excised and then coronally sliced into $200 \mu \mathrm{m}$ sections by VF-200 Slicer (Precisionary Instruments INC, Greenville, NC, USA). Bilateral hippocampus were isolated and then incubated with $0.125 \%$ trypsin. Hippocampus were triturated and suspended in DMEM medium (Invitrogen, Carlsbad, CA, USA) to prepare cell suspension. The suspension was then filtered through a $60 \mu \mathrm{m}$ nylon screen. Cell pellets were harvested and cultured in DMEM medium containing $1 \%$ Gluta Max, $1 \%$ sodium pyruvate and $10 \%$ fetal bovine serum. Four hours later, the culture medium was replaced by eurobasal medium with $1 \%$ Gluta Max and $2 \%$ B27. The neurons were cultured at $37{ }^{\circ} \mathrm{C}$ for 4 to 6 days before functional assays.

\subsection{Cell treatment and transfection}

For sevoflurane exposure, the isolated hippocampal neurons were exposed to $1.8 \%$ sevoflurane (Sigma Aldrich, St. Louis, MO, USA) for 6, 12, 24 or 48 hours before functional analysis. In multiple exposure groups, the neurons were pre-transfected with siRNA targeting CD82 (siCD82) (GenePharma, Suzhou, China) or co-transfected with siTRPM7 (GenePharma) and pcDNA 3.1-CD82 (Invitrogen) via Lipofectamine LTX (Invitrogen). Twenty-four hours later, the neurons were exposed to $1.8 \%$ sevoflurane for 12 hours before functional analysis. 

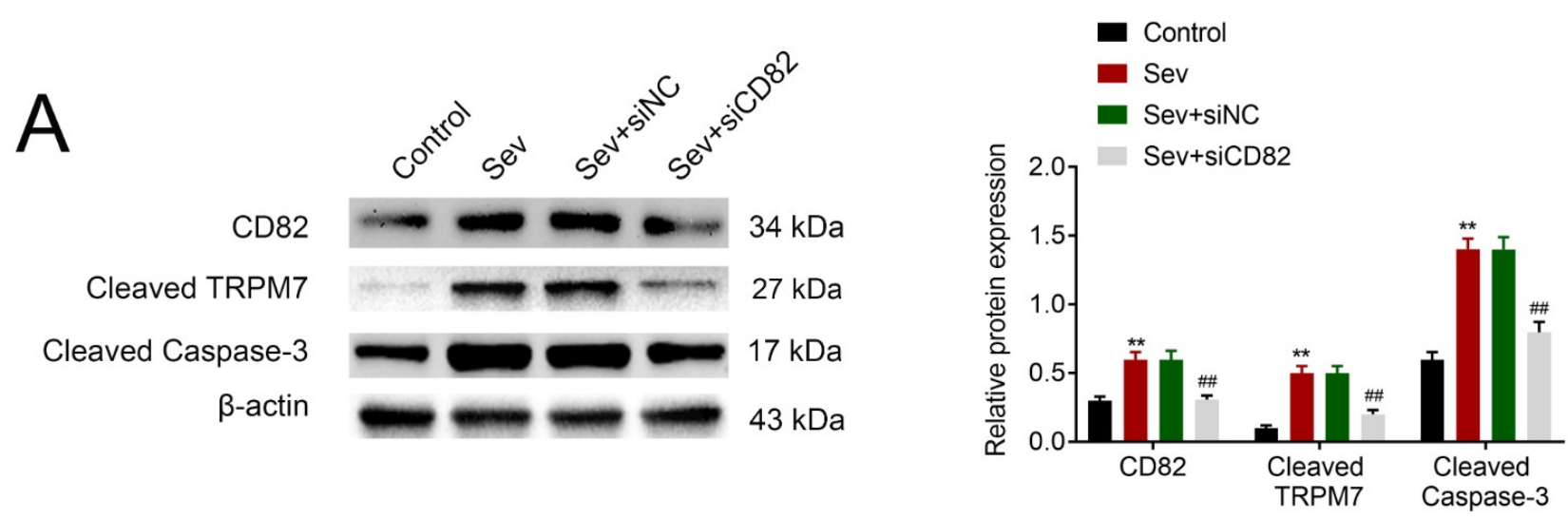

F I G U R E 3. Sevoflurane promoted cleavage of TRPM7 through enhance of CD82.

Pre-transfection with siCD82 attenuated sevoflurane-induced increase oin CD82, cleaved TRPM7 and cleaved caspase 3 expression. **, \#\# $p<0.01$.

\subsection{Cell viability}

After indicated treatment, neurons were plated and incubated with $1 \mathrm{mg} / \mathrm{mL}$ MTT solution (Sigma Aldrich) for 4 hours at 37 ${ }^{\circ} \mathrm{C}$. Formazan crystals in each well were dissolved by dimethyl sulfoxide, and the OD values at $490 \mathrm{~nm}$ were recorded.

\subsection{Flow cytometry}

After indicated treatment, neurons were treated with 5 $\mu \mathrm{L}$ propidium iodide and $5 \mu \mathrm{L}$ Annexin V-fluorescein isothiocyanate (70-AP101-100-AVF, MultiSciences, Hangzhou, China). Flow cytometer (BD Biosciences, San Jose, CA, USA) was then used for the analysis of cell apoptosis and the cell apoptotic rate.

\subsection{Western blot}

Proteins from neurons were extracted by RIPA buffer (Beyotime Institute of Biotechnology, Beijing, China). The concentration was determined by bicinchoninic acid assay kit (Pierce Biotechnology, Rockford, IL, USA). For western blot analysis, protein samples $(30 \mu \mathrm{g})$, separated by SDS-PAGE, were transferred onto polyvinylidene fluoride membrane (Merck Millipore, Billerica, MA, USA). Membrane was blocked with 5\% skim milk, followed by incubation with primary antibodies, including anti-CD82 (1 : 2000, Abcam, Cambridge, UK), antiCleaved TRPM7 (1 : 2500, Abcam), anti-Cleaved caspase3 (1: 3000, Abcam) and anti- $\beta$-actin (1: 3500, Abcam). Horseradish peroxidase-labled second antibodies were then incubated, and the bands were visualized using ECL Western blotting detection kits (Millipore, Billerica, MA, USA). The densitometry of the bands was quantified by Image J $1.38 \mathrm{X}$ software.

\subsection{Statistical analysis}

Data were expressed as means \pm SEM, and all the experiments were repeated for at least three times. Statistical analysis was performed by one-way ANOVA. p value less than 0.05 was considered statistically significant.

\section{Results}

\subsection{Expression of CD82 and TRPM7 in sevoflurane-induced neurons}

Hippocampal neurons were isolated from neonatal rats (postnatal day 1 or 2 ), and then exposed to $1.8 \%$ sevoflurane for $6,12,24$ or 48 hours. Cell viability of neurons was decreased time dependently by sevoflurane exposure (Fig. 1A). Protein expressions of CD82 and TRPM7 were increased by sevoflurane exposure (Fig. 1B) in a time-dependent manner, suggesting the potential regulatory ability of CD82/TRPM7 on sevoflurane-induced neurotoxicity.

\subsection{Knockdown of CD82 attenuated sevoflurane-induced neurotoxicity}

Sevoflurane decreased the cell viability in neurons (Fig. 2A), while pre-transfection with siCD82 attenuated the inhibitory effect of sevoflurane on neuronal viability (Fig. 2A). Moreover, cell apoptosis of neurons was promoted followed sevoflurane treatment (Fig. 2B). However, knockdown of CD82 reversed sevoflurane-induced promoting of cell apoptosis (Fig. 2B), indicating that knockdown of CD82 attenuated sevoflurane-induced neurotoxicity.

\subsection{Sevoflurane promoted cleavage of TRPM7 through enhance of CD82}

Western blot analysis demonstrated that sevoflurane treatment increased the expression of cleaved TRPM7 and cleaved caspase 3 (Fig. 3). However, the increase in cleaved TRPM7 and cleaved caspase 3 were attenuated in neurons pre-transfected with siCD82 (Fig. 3), indicating that sevoflurane promoted cleavage of TRPM7 through enhance of CD82.

\subsection{CD82 aggravated sevoflurane-induced neurotoxicity through cleavage of TRPM7}

To evaluate the role of CD82/TRPM7 in sevoflurane-induced neurotoxicity in the developing neurons, neurons were cotransfected with pcDNA-CD82 and siTRPM7, and then ex- 

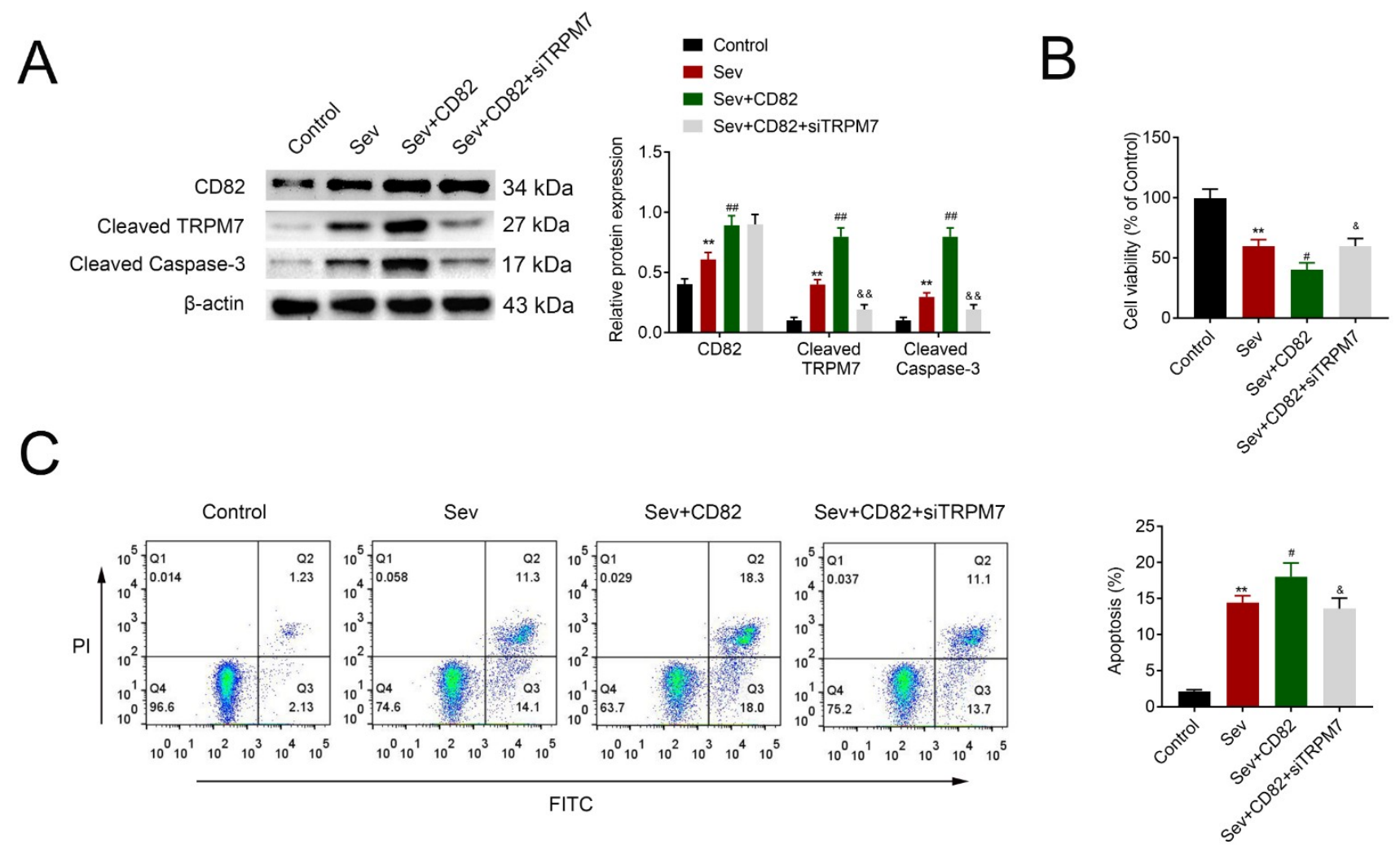

F I G U R E 4. CD82 aggravated sevoflurane-induced neurotoxicity through cleavage of TRPM7.

(A) Over-expression of CD82 enhanced expression of CD82, cleaved TRPM7 and cleaved caspase 3 in sevoflurane-induced neurons. Knockdown of TRPM7 attenuated CD82 over-expression-induced increase of cleaved TRPM7 and cleaved caspase 3. (B) Forced CD82 expression aggravated sevoflurane-induced neurotoxicity with decreased cell viability, while knockdown of TRPM7 counteracted with the promotive effect of CD82 on sevoflurane-induced neurotoxicity through increase of cell viability. (C) Forced CD82 expression aggravated sevoflurane-induced neurotoxicity with increased cell apoptosis, while knockdown of TRPM7 counteracted with the promotive effect of CD82 on sevoflurane-induced neurotoxicity through decrease of cell apoptosis. $\#, \& p<0.05, * *, \#, \& \& p<0.01$.

posed to sevoflurane. Over-expression of CD82 enhanced the expression of CD82, cleaved TRPM7 and cleaved caspase 3 in sevoflurane-induced neurons (Fig. 4A). However, the increased cleaved TRPM7 and cleaved caspase 3 in sevofluraneinduced neurons transfected with pcDNA-CD82 were reversed by additional transfection with siTRPM7 (Fig. 4A), confirming that sevoflurane promoted cleavage of TRPM7 through the enhanced expression of CD82. Moreover, forced CD82 expression aggravated sevoflurane-induced neurotoxicity with decreased cell viability (Fig. 4B) and increased cell apoptosis (Fig. 4C). However, knockdown of TRPM7 counteracted the promoting effect of CD82 on sevoflurane-induced neurotoxicity through the increase in cell viability (Fig. 4B) and decrease in cell apoptosis (Fig. 4C), suggesting that CD82 aggravated sevoflurane-induced neurotoxicity through cleavage of TRPM7 in the developing neurons.

\section{Discussion}

Ions, including $\mathrm{Ca}^{2+}$ and $\mathrm{Mg}^{2+}$, are important for brain development [14], and overload of the ions via ion channels could contribute anesthesia-induced neurotoxicity [14].
TRPM7 traces metal ions to maintain cell metabolism [10], and blockers or RNA interference for TRPM7 have been shown to attenuate $\mathrm{Zn}{ }^{2 \square}$-induced neurotoxicity [12] and protect hippocampal neurons against ischemia-induced cell death [15]. Since TRPM7 was cleaved by CD82 during agerelated cognitive impairment [9], the role of CD82/TRPM7 axis in sevoflurane-induced neurotoxicity in the developing neurons was then validated in this study.

Preclinical study has shown that children exposed to sevoflurane demonstrated higher apoptosis status of neural stem cells [16]. Moreover, anesthetic sevoflurane could also induce neurotoxicity in neuronal cells with decreased cell viability and increased cell apoptosis [17]. The data from this study showed that exposure of the isolated neurons from neonatal rats to sevoflurane induced severely neurotoxicity with decreased cell viability and increased cell apoptosis. Furthermore, CD82 and TRPM7 were increased in neurons post sevoflurane exposure, suggesting the regulatory role of CD82/TRPM7 on sevoflurane-induced neurotoxicity in the developing neurons.

Functional assays indicated that forced CD82 aggravated sevoflurane-induced neurotoxicity with increased cell viability 
and decreased cell apoptosis of neurons, while knockdown of CD82 decreased the cell viability and increased the cell apoptosis, therebt alleviating sevoflurane-induced neurotoxicity. In addition, TRPM7 silencing attenuated CD82 over-expressioninduced neurotoxicity in sevoflurane-induced neurons, demonstrating that CD82 might aggravate sevoflurane-induced neurotoxicity through regulation of TRPM7. Antioxidant defense was increased in children exposed to sevoflurane [16], and levels of reactive oxygen species were also accumulated in neonatal rats post sevoflurane exposure [4]. The reduction of reactive oxygen species was shown to be helpful for the amelioration of sevoflurane-induced neurotoxicity [17]. TRPM7 could release $\mathrm{Zn}^{2+}$ through sensation of oxidative stress [18]. Therefore, whether CD82/TRPM7 could regulate oxidative stress in neurons to aggravate sevoflurane-induced neurotoxicity should be further investigated. Moreover, exposure of sevoflurane was reported to induce autophagy of hippocampal neurons [19]. CD82 was required for the interaction with Tolllike receptor 9 to dictate autophagy [20] and TRPM7-mediated $\mathrm{Ca}^{2+}$ influx could regulate basal autophagy [21]. Therefore, CD82/TRPM7 might also regulate autophagy in sevofluraneinduced neurons to aggravate the neurotoxicity.

Sevoflurane exposure has been shown to elevate caspase3 activation [4], and caspase 3 could promote cleavage of TRPM7 for disassociation of the $\alpha$-kinase domain, thus participating in cell apoptosis [22]. Over-expression of CD82 promoted TRPM7 $\alpha$-kinase cleavage through activation of caspase 3 [9]. Western blot analysis in this study demonstrated that forced CD82 expression in neurons increased the protein expression of cleaved TRPM7 and cleaved caspase 3, while knockdown of CD82 decreased the protein expression. Moreover, knockdown of TRPM7 attenuated CD82 overexpression-induced increase in cleaved TRPM7 and cleaved caspase 3, suggesting that CD82 might aggravate sevofluraneinduced neurotoxicity through TRPM7 cleavage. Additionally, since suppression of TRPM7 could induce cell death of gastric cancer [23], the effects of TRPM7 on cell viability and apoptosis of sevoflurane-induced neurons should be investigated in the further study. Administration of RNA interference of CD82 and TRPM7 in neonatal rats might be performed to provide in vivo evidence for the regulatory role of CD82/TRPM7 on sevoflurane-induced neurotoxicity.

\section{Conclusions}

This study demonstrated that enhanced CD82 and TRPM7 were related to sevoflurane-induced neurotoxicity. CD82 promoted the cleavage of TRPM7, resulting in up-regulation of CD82 and cleaved TRPM7 in hippocampus of developing brains. In addition, pretreatment with siCD82 decreased cleaved TRPM7 and cleaved caspase 3 in neurons and mitigated sevoflurane-induced decrease in cell viability and increase in cell apoptosis, thereby ameliorating sevofluraneinduced neurotoxicity. These findings proposed that sevoflurane-induced neurotoxicity in the developing brains could be attenuated by knockdown of CD82.

\section{ACKNOWLEDGEMENTS}

Thanks to all the peer reviewers and editors for their opinions and suggestions.

\section{CONFLICT OF INTEREST}

The authors state that there are no conflicts of interest to disclose.

\section{FUNDING}

This work was supported by the Fundamental Research Funds for the Central Universities (Grant No. xzy012019121).

\section{ETHICS APPROVAL}

All animal experiments were approved by the Biomedical Ethics Committee of Ninth Hospital of Xi'an (Approval no.2020-046) for the use of animals and conducted in accordance with the National Institutes of Health Laboratory Animal Care and Use Guidelines.

\section{STATEMENT OF INFORMED CONSENT}

Not applicable.

\section{AVAILABILITY OF DATA AND MATERIALS}

All data generated or analyzed during this study are included in this published article.

\section{AUTHORS' CONTRIBUTIONS}

Qing Shu designed the study, supervised the data collection, Xiaoyan Zhao analyzed the data, interpreted the data, Xin Geng and Xiaoye Wang prepare the manuscript for publication and reviewed the draft of the manuscript. All authors have read and approved the manuscript.

\section{REFERENCES}

[1] Fredriksson A, Ponten E, Gordh T, et al. Neonatal exposure to a combination of N-methyl-D-aspartate and gamma-aminobutyric acid type A receptor anesthetic agents potentiates apoptotic neurodegeneration and persistent behavioral deficits. Anesthesiology. 2007;107:427-436.

[2] Chen B, Deng X, Wang B, et al. Persistent neuronal apoptosis and synaptic loss induced by multiple but not single exposure of propofol contribute to long-term cognitive dysfunction in neonatal rats. J Toxicol Sci. 2016;41:627-636.

[3] Sprung J, Flick RP, Katusic SK, et al. Attention-deficit/hyperactivity disorder after early exposure to procedures requiring general anesthesia. Mayo Clin Proc. 2012;87:120-129.

[4] Liu B, Gu Y, Xiao H, et al. Altered Metabolomic Profiles May Be Associated with Sevoflurane-Induced Neurotoxicity in Neonatal Rats. Neurochemical Research. 2015;40:788-799.

[5] Termini CM, Cotter ML, Marjon KD, et al. The membrane scaffold CD82 regulates cell adhesion by altering alpha4 integrin stability and molecular density. Mol Biol Cell. 2014;25:1560-1573.

[6] Wang Y, Chen H, Fu Y, et al. MiR-195 inhibits proliferation and growth 
and induces apoptosis of endometrial stromal cells by targeting FKN. Int J Clin Exp Pathol. 2013;6:2824-2834.

[7] Mela A, Goldman JE. CD82 blocks cMet activation and overcomes hepatocyte growth factor effects on oligodendrocyte precursor differentiation. J Neurosci. 2013;33:7952-7960.

[8] Rojas-Hernandez CM, Nemunaitis J, Marjon KD, et al. Chronic lymphocytic leukemia with clinical debut as neurological involvement: a rare phenomenon and the need for better predictive markers. BMC hematology. 2017;17:3-3.

[9] Zhao Y, Kiss T, DelFavero J, et al. CD82-TRPM7-Numb signaling mediates age-related cognitive impairment. Geroscience. 2020;42:595611.

[10] Park HS, Hong C, Kim BJ, et al. The Pathophysiologic Roles of TRPM7 Channel. The Korean journal of physiology \& pharmacology : official journal of the Korean Physiological Society and the Korean Society of Pharmacology. 2014;18:15-23.

[11] Abumaria N, Li W, Clarkson AN. Role of the chanzyme TRPM7 in the nervous system in health and disease. Cell Mol Life Sci. 2019;76:33013310.

[12] Kim Y, Oh HG, Cho YY, et al. Stress hormone potentiates $\mathrm{Zn}(2+)$ induced neurotoxicity via TRPM7 channel in dopaminergic neuron. Biochem Biophys Res Commun. 2016;470:362-367.

[13] Chen W, Xu B, Xiao A, et al. TRPM7 inhibitor carvacrol protects brain from neonatal hypoxic-ischemic injury. Mol Brain. 2015;8:11.

[14] $\mathrm{Wu} \mathrm{J,} \mathrm{Yang} \mathrm{JJ,} \mathrm{Cao} \mathrm{Y,} \mathrm{et} \mathrm{al.} \mathrm{Iron} \mathrm{overload} \mathrm{contributes} \mathrm{to} \mathrm{general}$ anaesthesia-induced neurotoxicity and cognitive deficits. J Neuroinflammation. 2020;17:110.

[15] Sun HS, Jackson MF, Martin LJ, et al. Suppression of hippocampal TRPM7 protein prevents delayed neuronal death in brain ischemia. Nat Neurosci. 2009;12:1300-1307.

[16] Zhou X, Lu D, Li WD, et al. Sevoflurane Affects Oxidative Stress and
Alters Apoptosis Status in Children and Cultured Neural Stem Cells. Neurotox Res. 2018;33:790-800.

[17] Qi J, Jia Y, Wang W, et al. The role of Bag2 in neurotoxicity induced by the anesthetic sevoflurane. J Cell Biochem. 2018.

[18] Abiria SA, Krapivinsky G, Sah R, et al. TRPM7 senses oxidative stress to release $\mathrm{Zn}(2+)$ from unique intracellular vesicles. Proceedings of the National Academy of Sciences of the United States of America. 2017;114:E6079-E6088.

[19] Xu L, Shen J, Yu L, et al. Role of autophagy in sevofluraneinduced neurotoxicity in neonatal rat hippocampal cells. Brain Res Bull. 2018;140:291-298.

[20] Khan NS, Lukason DP, Feliu M, et al. CD82 controls CpG-dependent TLR9 signaling. FASEB journal : official publication of the Federation of American Societies for Experimental Biology. 2019;33:12500-12514.

[21] Oh HG, Chun YS, Park CS, et al. Regulation of basal autophagy by transient receptor potential melastatin 7 (TRPM7) channel. Biochem Biophys Res Commun. 2015;463:7-12.

[22] Desai BN, Krapivinsky G, Navarro B, et al. Cleavage of TRPM7 releases the kinase domain from the ion channel and regulates its participation in Fas-induced apoptosis. Developmental cell. 2012;22:1149-1162.

[23] Kim BJ, Park EJ, Lee JH, et al. Suppression of transient receptor potential melastatin 7 channel induces cell death in gastric cancer. Cancer Sci. 2008;99:2502-2509.

How to cite this article: Qing Shu, Xiaoyan Zhao, Xin Geng, Xiaoye Wang. CD82 Aggravates Sevoflurane - Induced Neurotoxicity by Regulating TRPM7 in Developing Neurons. Signa Vitae. 2020;16(2):142-147. doi:10.22514/sv.2020.16.0066. 